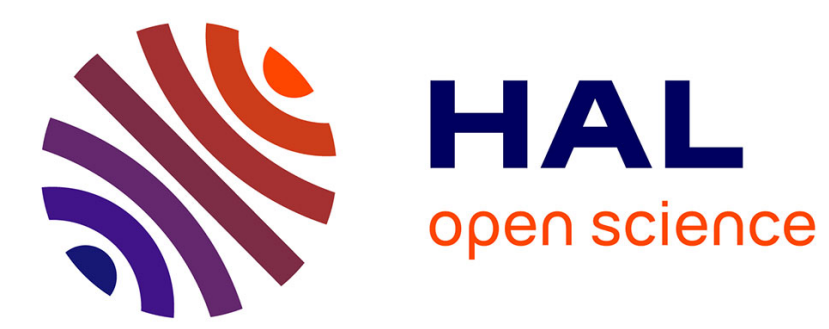

\title{
R-MATRIX METHOD - ADVANTAGES AND APPLICATIONS
}

P. Burke

\section{To cite this version:}

P. Burke. R-MATRIX METHOD - ADVANTAGES AND APPLICATIONS. Journal de Physique Colloques, 1978, 39 (C4), pp.C4-27-C4-34. 10.1051/jphyscol:1978404 . jpa-00217438

\section{HAL Id: jpa-00217438 https://hal.science/jpa-00217438}

Submitted on 1 Jan 1978

HAL is a multi-disciplinary open access archive for the deposit and dissemination of scientific research documents, whether they are published or not. The documents may come from teaching and research institutions in France or abroad, or from public or private research centers.
L'archive ouverte pluridisciplinaire HAL, est destinée au dépôt et à la diffusion de documents scientifiques de niveau recherche, publiés ou non, émanant des établissements d'enseignement et de recherche français ou étrangers, des laboratoires publics ou privés. 


\title{
R-MATRIX METHOD - ADVANTAGES AND APPLICATIONS
}

\author{
P. G. BURKE
}

Department of Applied Mathematics and Theoretical Physics, Queen's University, Belfast BT7 1NN, N. Ireland, U.K.

and

Daresbury Laboratory, Science Research Council, Daresbury, Warrington WA4 4AD, U.K.

\begin{abstract}
Résumé. - Les concepts fondamentaux de la méthode de la matrice $R$ sont exposés ainsi que les développements théoriques récemment survenus. Les résultats relatifs à l'affinité électronique, la diffusion des électrons par les atomes et les ions et la photo-ionisation des atomes sont ensuite présentés.
\end{abstract}

\begin{abstract}
The basic concept of the $R$-matrix method is discussed and recent theoretical developments are reviewed. Some results are then presented for electron affinities, electron scattering by atoms and ions and photoionisation of atoms.
\end{abstract}

1. Introduction. - The $R$-matrix method was first introduced by Wigner [1] and Wigner and Eisenbud [2] in a study of nuclear reactions. In suosequem years the theory was considerably extended by many others workers in nuclear physics and comprehensive reviews written by Lane and Thomas [3] and by Breit [4]. Over the last eight years the method has been further developed to enable it to describe a broad range of atomic and molecular continuum processes and results have been obtained for electron affinities, electron scattering by atoms ions and molecules, atomic polarizabilities and Van der Waals coefficients, photoionization of atoms and ions and non-linear optical coefficients. In addition consideration is now being given to applying the method to free-free transitions, photo-detachment and dissociative recombination and attachment. A review of some of these applications in atomic physics has been given by Burke and Robb [5].

2. Review of the theory. - The essential idea of the method is that configuration space describing the scattered or ejected electron and the target atom or molecule is divided into two regions as illustrated in figure 1 . The internal region is defined by a sphere of radius a such that the charge distribution of the target states of interest are just contained within the sphere. In some situations for example when electron molecule collisions are being considered in prolate spheroidal coordinates a more complex surface may be appropriate but the essential idea remains unaltered.

In the internal region where the scattered electron has penetrated the charge distribution of the target,

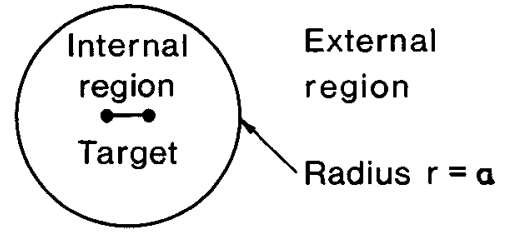

FIG. 1. - The internal and external regions in $R$-matrix theory.

the interaction is strong with both electron exchange and correlation effects important. However in the external region exchange between the scattered electron and the target can clearly be neglected, and the collision is dominated by the Coulomb and/or centrifugal terms and multipole terms in the interaction. The Schrödinger equation then has an analytic solution or one that can relatively easily be obtained by numerical methods.

In the internal region the problem is very similar to a standard bound state calculation and consequently in all applications that have so far been made a configuration interaction expansion of the following type has been adopted

$$
\Psi_{k}=\mathcal{A} \sum_{i j} a_{i j k} \Phi_{i} u_{j}^{0}(r)+\sum_{j} b_{j k} \varphi_{j} .
$$

In this equation the $\Phi_{i}$ are functions representing the target states of interest coupled with functions representing the angular and spin motion of the scattered electron. The target states themselves are represented by $\mathrm{CI}$ wave functions constructed from a bound orbital basis. The $u_{j}^{0}$ are continuum basis orbitals representing the scattered electron and conse- 
quently, unlike the bound orbital basis, they do not vanish on the boundary. $\mathcal{A}$ is the usual antisymmetrization operator which ensures that the first expansion is totally antisymmetric. The $\varphi_{j}$ are $(N+1)$ - electron correlation functions constructed from the bound orbitals. These functions have a dual sole. Firstly they are included to ensure that the total wave function is complete when the continuum orbital basis is orthogonal to the bound orbital basis. Secondly they can allow for further electron correlations beyond those included in the first expansion. Finally the coefficient $a_{i, k}$ and $b_{j k}$ are coefficients which are determined by diagonalizing the Hamiltonian as discussed below.

The information obtained from the calculation in the internal region can be expressed as an $R$-matrix on the surface bounding this region. If we impose logarithmic boundary conditions on the surface of the internal region defined by the equation

$$
\left.\frac{\mathrm{d} u_{i k}}{\mathrm{~d} r}\right|_{r=a}=b_{i} u_{i k}(a)
$$

where the $u_{i k}$ are the radial functions in equation (1) defined by

$$
u_{i k}(r)=\sum_{j} a_{i j k} u_{j}^{0}(r)
$$

then the continuum breaks up into a discrete set of energies $E_{k}, k=1,2, \ldots, \infty$. The corresponding eigenstates $\Psi_{k}$ from a complete set in the internal region which can be used as a basis for the expansion of the wave function $\Psi_{E}$ in this region for any energy $E$

$$
\Psi_{E}=\sum_{k} A_{E_{k}} \Psi_{k}
$$

By substituting this expansion into the Schrödinger equation

$$
(\mathfrak{H}-E) \Psi_{k}=0
$$

and using the boundary condition (2), we find directly that the logarithmic derivative of the radial wave function describing the scattered electron at energy $E$ is

$$
u_{i E}(a)=\sum_{j} R_{i j}(E)\left(a \frac{\mathrm{d} u_{j E}}{\mathrm{~d} r}-b_{j} u_{j E}\right)_{r=a}
$$

where

$$
R_{i j}(E)=\frac{1}{2 a} \sum_{k} \frac{u_{i k}(a) u_{j k}(a)}{E_{k}-E}
$$

is the $R$-matrix.

The basic problem in the $R$-matrix method then is to calculate the eigenfunctions $\Psi_{k}$ and the corresponding eigenenergies $E_{\mathrm{k}}$ which define the $R$-matrix and which give a complete description of the collision problem in the internal region. The solution of the problem in the external region then starts from equation (6), which defines the boundary condition on the surface, and then by integrating out to infinity the phase shift and cross section is determined.

We now turn to a brief discussion of approaches which have been used to calculate the eigenfunctions $\Psi_{k}$ and the eigenenergies $E_{k}$. In the first, adopted by Burke et al. [7] for atoms, the Hamiltonian is divided into two parts

$$
\mathscr{H}=\mathscr{H}_{0}+\mathfrak{H}_{\text {int }}
$$

where $\mathscr{H}_{0}$ is a zero-order potential problem which can be solved numerically subject to the boundary conditions (2) to yield the continuum basis orbital $u_{j}^{0}$. The coefficients $a_{i j k}$ and $b_{j k}$ are then determined by diagonalizing the Hamiltonian

$$
\left\langle\Psi_{k}|\mathfrak{H}| \Psi_{k^{\prime}}\right\rangle=E_{k} \delta_{k k^{\prime}} .
$$

An important advantage of this approach is that this single diagonalization determines all the $\Psi_{k}$ and thus completely determines the solution in the inner region for all energies. However a correction determined by solving the zero-order problem [8] has to be included for the high lying omitted states in the expansion.

In the second approach adopted by Fano and Lee [9] and Lee [10] the boundary condition defined by equation (2) satisfied by the basis orbitals $u_{j}^{0}$ is adjusted so that an eigenvalue $E_{k}$ falls at the energy $E$ of interest. In practise $n$ such eigenvalues must be found where $n$ is the number of channels. In this case the $R$-matrix is determined by just the singular terms in its expansion and no Buttle correction is required. In practise this means that there is no longer any need to define a zero-order problem as in the previous method and consequently analytic STO's or GTO's can be used for the continuum basis orbitals. However this method does require several diagonalization of the Hamiltonian matrix at each energy of interest, although the resultant wave function then has a continuous derivative on the boundary.

The third approach which is now being actively persued for electron molecule scattering by Schneider [11], Schneider and Hay [12] and Burke et al. [13] combines the advantages of using analytic continuum basis orbitals and a single diagonalization of the Hamiltonian. The continuum basis orbitals used in this case are either STO's or GTO's and satisfy arbitrary boundary conditions on the surface of the internal region which means that the Hamiltonian matrix is not symmetric due to non-cancelling surface terms. However if the Block operator $L_{\mathrm{b}}[14]$ defined by

$$
L_{\mathrm{b}}=\frac{1}{2} \sum_{i}\left|\Phi_{i}\right\rangle \delta(r-a)\left(\frac{\mathrm{d}}{\mathrm{d} r}-\frac{b_{i}}{a}\right)\left\langle\Phi_{i}\right|
$$

is added to $\mathcal{H}$ the resultant operator is symmetric. The $R$-matrix basis states are thus determined by diagonalizing $\mathscr{H}+L_{\mathrm{b}}$ rather than $\mathscr{H}$ as in equa- 
tion (9). The contribution of the high lying states is not so important as in the first approach mentioned above and a Buttle type correction is not required.

Further approaches have recently been discussed by Shimamura [15] which might have advantages but so far no detailed calculations for real atomic and molecular systems have been carried out.

To conclude this brief review of the theory we emphasis again that the basic expansion (1) in the internal region is very closely analogous to that used in bound state problems. As a result those approaches which use analytic continuum basis orbitals can use bound state computer codes with very little modification. It is this aspect of the $R$-matrix method which may be most important in the proposed extensions to treat problems involving polyatomic molecules where otherwise the investment in new computer software would be very great indeed.

3. Recent results. - In this section we present some recent applications. These have all been obtained using a general computer code based on the first approach [16].

3.1 Electron attaChment enERgies. - Expansion (4) can be used to calculate bound state wave functions and energies. In this case it is only necessary to find by iteration that solution in the external region for which that wave functions decays exponentially in all channels. Le Dourneuf and Vo $\mathrm{Ky}$ Lan [17] have recently used the $R$-matrix method to obtain accurate $a b$ initio results for the attachment energies of $\mathrm{C}, \mathrm{N}$ and $\mathrm{O}$. In their calculation they included in expansion (1) all three terms of the ground state configuration as well as polarized pseudo-states which give correctly the long range polarization potential which is particularly important for these weakly bound systems. Their results are compared with experiment and with some other theories in table I.

From this table the limitations of the standard structure methods, such as Hartree-Fock, BetheGoldstone and Superposition of Configurations are obvious. For example, the most extensive SOC calculation of Sasaki and Yoshimine, which includes as many as 2649 configurations for $\mathrm{O}^{-}$, has an error of $0.33 \mathrm{eV}$ for $\mathrm{O}^{-}$and nearly $0.5 \mathrm{eV}$ for $\mathrm{N}^{-}$. The latter state is now thought to lie in the continuur and to give rise to a large low energy peak in th electron scattering cross section (see Fig. 3). Th $R$-matrix method, which is basically a collisiona approach, focuses directly on the energy differenc between the target ground state and the negativ ion state and in all cases is in reasonable agreemen with experiment.

3.2 EleCtron ATOM SCATTERING. - The $R$-matrix method has been used to calculate collision cross sections for a number of atoms including atomic hydrogen, helium, boron, neon as well as carbon, oxygen and nitrogen. We will present here results for the last three atoms which complement the attachment energy calculations described in the previous sub-section.

The $R$-matrix calculations were carried out using three approximations for the wave function expansion (1).

SC : This includes all terms of the ground state configuration $1 \mathrm{~s}^{2} 2 \mathrm{~s}^{2} 2 \mathrm{p}^{q}$.

$\mathrm{MC}$ : This includes all terms which correspond to the three configurations $1 \mathrm{~s}^{2} 2 \mathrm{~s}^{2} 2 \mathrm{p}^{q}, 1 \mathrm{~s}^{2} 2 \mathrm{~s} 2 \mathrm{p}^{q+1}$ and $1 \mathrm{~s}^{2} 2 \mathrm{p}^{q+2}$.

PS : This includes the three terms of the ground state configuration as for $\mathrm{SC}$ and also the polarized pseudo-states which allow for the full polarizability of the ground state. This approximation is essentially the same as that used for the attachment energy calculations discussed above.

We show in figure 2 the total cross section for electron scattering on the ground state for carbon. The SC calculation, which omits all of the polarization potential, gives too little attraction and the state of $\mathrm{C}^{-}$appears in the continuum giving rise to a spurious resonance. The PS and MC calculations are quite close giving a resonance at about $0.6 \mathrm{eV}$. The Bethe Goldstone calculations of Thomas and Nesbet [22] required an empirical correlation energy adjustment of 0.039 Ryd to reproduce the experimental position of the $C^{-}\left({ }^{2} D^{0}\right)$ state. With this adjustment the $\mathrm{BG}$ cross section curve is quite close to the PS and MC curves which are probably a fairly good indication of the exact cross section.

In figure 3 we show the total cross section for electron scattering on the ground state of nitrogen.

TABLE I

Attachment energies (in $\mathrm{eV}$ ) of carbon, nitrogen and oxygen atoms

\begin{tabular}{|c|c|c|c|c|}
\hline & ${ }^{4} S^{0}$ & ${ }^{2} \mathrm{D}^{0}$ & $\begin{array}{l}\mathbf{N}^{-} \\
{ }^{3} \mathrm{P}^{\mathrm{e}}\end{array}$ & $\begin{array}{l}\mathrm{O}^{-} \\
{ }^{2} \mathrm{P}^{0}\end{array}$ \\
\hline Experiment [18] & 1.262 & 0.035 & $-0.050 \pm 0.050 ?$ & 1.462 \\
\hline$R$-Matrix & 1.228 & 0.001 & $0 . \overline{0} 04$ & 1.412 \\
\hline $\mathrm{HF}$ [19] & 0.550 & & -2.150 & -0.542 \\
\hline $\mathrm{BG}[20]$ & 1.211 & -0.275 & -0.582 & 0.963 \\
\hline $\mathrm{SOC}[21]$ & 1.11 & & -0.52 & 1.13 \\
\hline
\end{tabular}




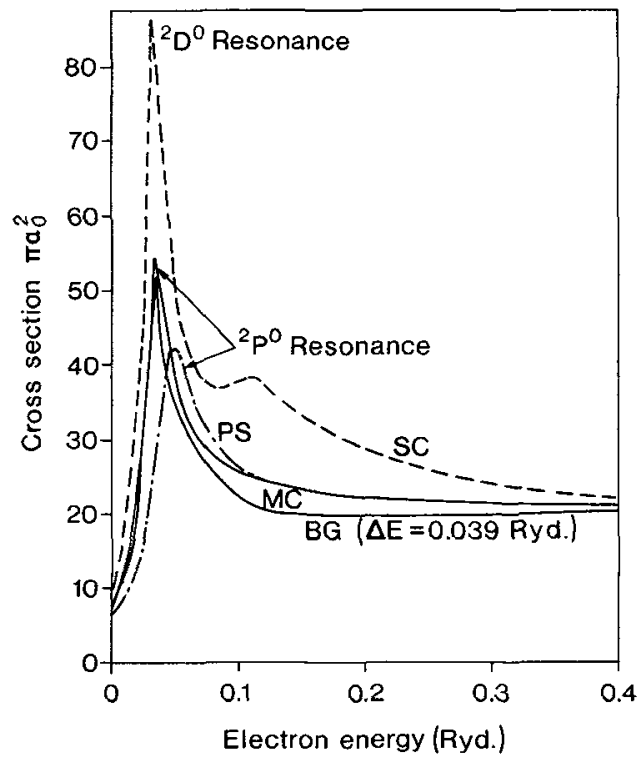

FIG. 2. - The total cross section for electron scattering on the ground state of carbon.

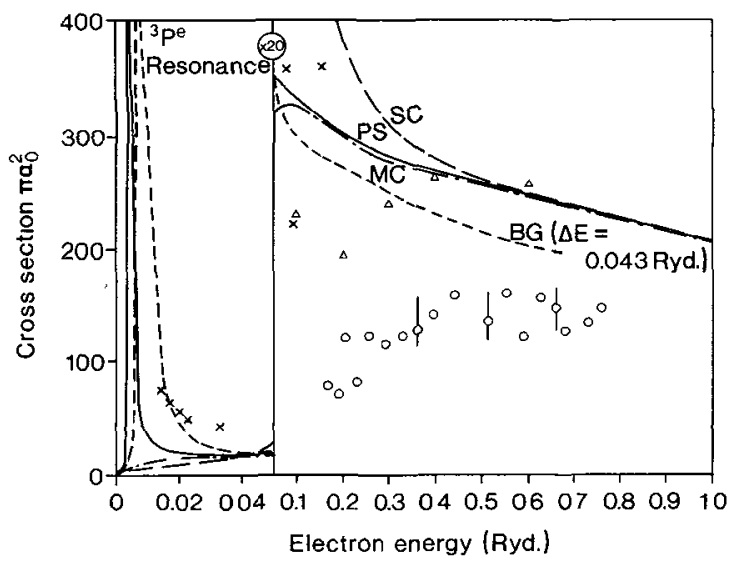

Fig. 3. - The total cross section for electron scattering on the ground state of nitrogen.

Again the SC calculation shows too little attraction while now the MC calculation probably overestimates the attraction. The PS calculation and the BG calculation (with an empirical adjustment of $0.043 \mathrm{Ryd}$ ) both give a low energy ${ }^{3} \mathrm{P}^{\mathrm{e}}$ resonance at about $50 \mathrm{meV}$ which seems in accord with experiments of Miller et al. [23]. (We note here that the PS calculations for the energy of this state reported in table I included more virtual excitations to excited pseudo-orbitals and lay some $50 \mathrm{meV}$ lower in energy.) However the final picture concerning the position of this resonance is still unclear and further experimental work is urgently needed. At higher energies the theories lie consistently above the experiments of Neynaber et al. [24] suggesting that some renormalization of the latter may be appropriate.

In figure 4 we also show the total cross section for electron scattering on the ground state of atomic

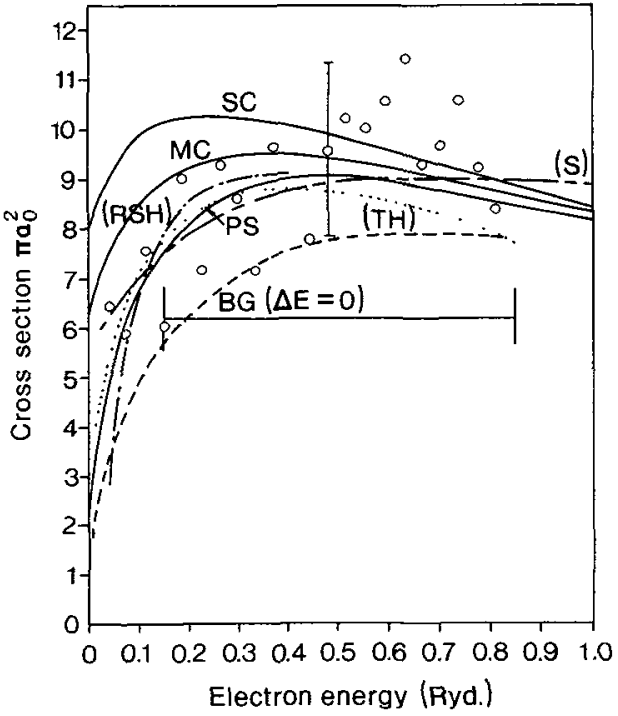

FIG. 4. - The total cross section for electron scattering on the ground state of oxygen.

oxygen. The experimental results of Lin and Kivel [25], Neynaber et al. [24] and Sunshine et al. [26] have large error bars and do not exclude any of the theoretical calculations. However it can be seen that the effect of the polarizability of the target is important with the PS results, including independent work by Rountree et al. [27], lying well below the SC results. The BG results may be too low at low energies but at higher energy the inclusion of more core correlation in this work may lead to a more accurate cross section.

Again new experimental measurements are urgently needed.

3. 3 EleCtRON ION SCATTERING. - A large program of work on electron ion scattering is being carried out using the $R$-matrix method because of the importance of these processes both in controlled thermonuclear fusion research and also in astrophysics. A recent review of same of this work has been given by Robb [28]. Here we will present some new results for Be-like ions which has proved particularly important in understanding the electron density in the solar transition region.

We show in figure 5 the first few energy levels in a typical ion in the Be-like isoelectronic sequence. An important line intensity ratio observed in the sun is

$$
R=\frac{I\left({ }^{3} \mathrm{P}^{\mathrm{e}} \rightarrow{ }^{3} \mathrm{P}^{0}\right)}{I\left({ }^{1} \mathrm{P}^{0} \rightarrow{ }^{1} \mathrm{~S}^{\mathrm{e}}\right)}
$$

This depends sensitively on the electron density. In order to calculate this dependence it is necessary to know the electron excitation cross sections involving these levels as well as the Einstein $A-$ coefficients for their decay. In a recent calculation [29] for $\mathrm{C}$ III and $\mathrm{O} V$ these cross sections were calculated including the $2 \mathrm{~s}^{2}{ }^{1} \mathrm{~S}^{\mathrm{e}}, 2 \mathrm{~s} 2 \mathrm{p}{ }^{3} \mathrm{p}^{0},{ }^{1} \mathrm{P}^{0}$ and $2 \mathrm{p}^{2}{ }^{3} \mathrm{P}^{\mathrm{e}}{ }^{1} \mathrm{D}^{\mathrm{e}}$ 


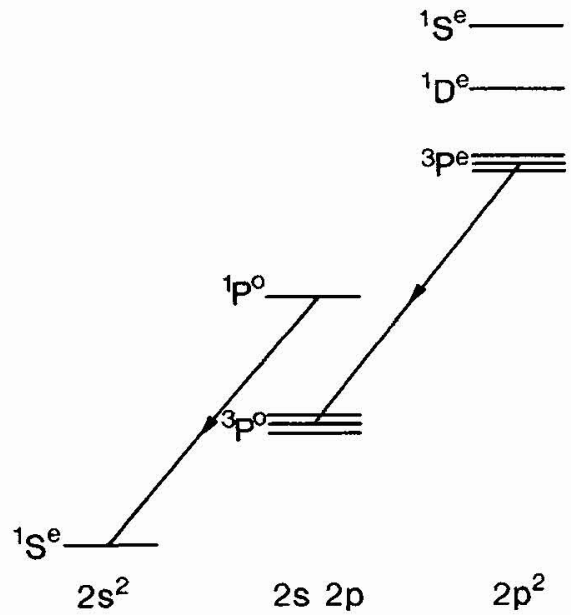

FiG. 5. - Energy level diagram of Be-like ions.

and ${ }^{1} S^{e}$ target eigenstates in expansion (1), where each of these states was represented as a linear combination of configurations including excitations to $\overline{3 \mathrm{~s}} \overline{3 \mathrm{p}} \overline{3 \mathrm{~d}}$ and $\overline{4 \mathrm{f}}$ pseudo-orbitals. As an indication of the results we show in figure 6 the total collision

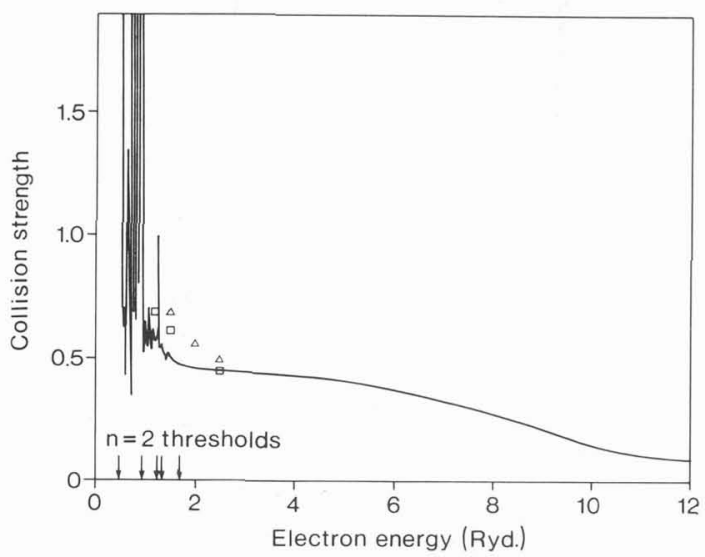

FIG. 6. - Collision strength for the $2 \mathrm{~s}^{2}{ }^{1} \mathrm{~S}^{\mathrm{e}}-2 \mathrm{~s} 2 \mathrm{p}^{3} \mathrm{P}^{0}$ transition in C III.

strength for the $2 s^{2}{ }^{1} \mathrm{~S}^{\mathrm{e}}-2 \mathrm{~s}^{2} \mathrm{p}{ }^{3} \mathrm{P}^{0}$ transition compared with earlier results of Eissner [30] and Flower and Launay [31] using less sophisticated approximations. The cross section at low energies is dominated by many Rydberg series of resonances converging to the higher $n=2$ thresholds. An advantage of the $R$-matrix method is that these are all calculated using a single diagonalization of the Hamiltonian. The $R$-matrix result for this transition was some $20 \%$ lower than previous work while that for the $2 s^{2}{ }^{1} S^{e}$ $2 \mathrm{~s} 2 \mathrm{p}{ }^{1} \mathrm{P}^{0}$ transition in C III was $20 \%$ higher. The effect on the line ratio $R$ is very significant as shown in figure 7. A quiet sun density of $3 \times 10^{9} \mathrm{~cm}^{-3}$ is deduced [32] in good agreement with other measurements, whereas the earlier transition strengths yielded a result an order of magnitude lower.

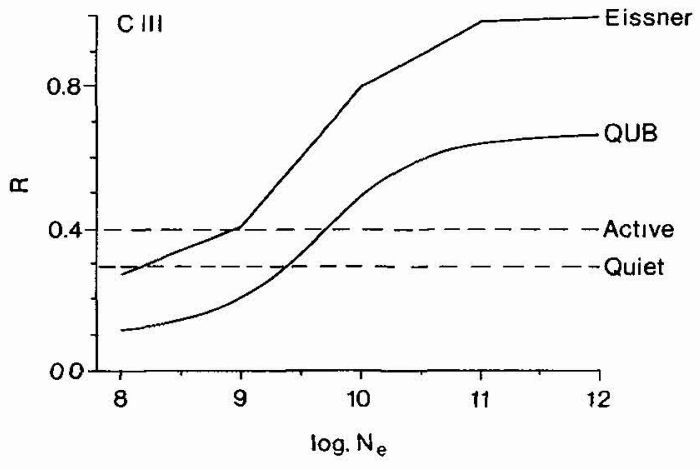

FIG. 7. - The dependence of the line ratio $R$ in C III on the electron density in the sun.

3.4 Photoionization of atoms. - One area where a very good comparison can be made with experiment is for the photoionization of atoms where many accurate data using synchrotron radiation sources are available. The matrix element which must be calculated in this case is

$$
M=\left\langle\Psi_{E_{f}}|\mathbf{D} \cdot \hat{\varepsilon}| \Psi_{E_{i}}\right\rangle
$$

where the initial state is a bound state of the electron ion system and the final state is a scattering state. In the $R$-matrix method both of these states are expanded using equation (4) where the same basis in terms of target ion states is used in expansion (1).

We consider first some results obtained for argon [33]. This atom is of importance since, as a closed shell system, it is particularly amenable to study by other theoretical approaches such as the Random Phase Approximation with Exchange (R.P.A.E.) [34] and the Many Body Perturbation theory (M.B.P.T.) [35]. The processes of main interest here involve the ejection of a $3 p$ or a $3 \mathrm{~s}$ electron

$$
h v+\mathrm{A}\left(3 \mathrm{~s}^{2} 3 \mathrm{p}^{6}{ }^{1} \mathrm{~S}^{\mathrm{e}}\right) \longrightarrow \mathrm{A}^{+}\left(3 \mathrm{~s}^{2} 3 \mathrm{p}^{5}{ }^{2} \mathrm{P}^{0}\right)+\mathrm{e}^{-}(k \mathrm{~s}, k \mathrm{~d})
$$

In the $R$-matrix calculations which have been carried out the two states of $\mathrm{A}^{+}$are retained in expansion (1). and two representations of these states considered. In the first they are represented by single configuration (SC) while in the second extensive configuration interaction (C I) is allowed by including double excitation into $\overline{3 \mathrm{~d}}$ and single excitation in to $\overline{4 \mathrm{~s}}$ and $\overline{4 \mathrm{p}}$ pseudo-orbitals. The results for the total cross section is given in figure 8 where they are compared with R.P.A.E. calculations of Amusia et al. [36] and the experiment of Marr and West [37]. The $R$-matrix length results are usually more accurate than the velucity since in equation (12) both the initial and final states are treated by a collisional wave function which has the correct asymptotic form. We see in this figure that the $\mathrm{CI}$ length results are in reasonable 


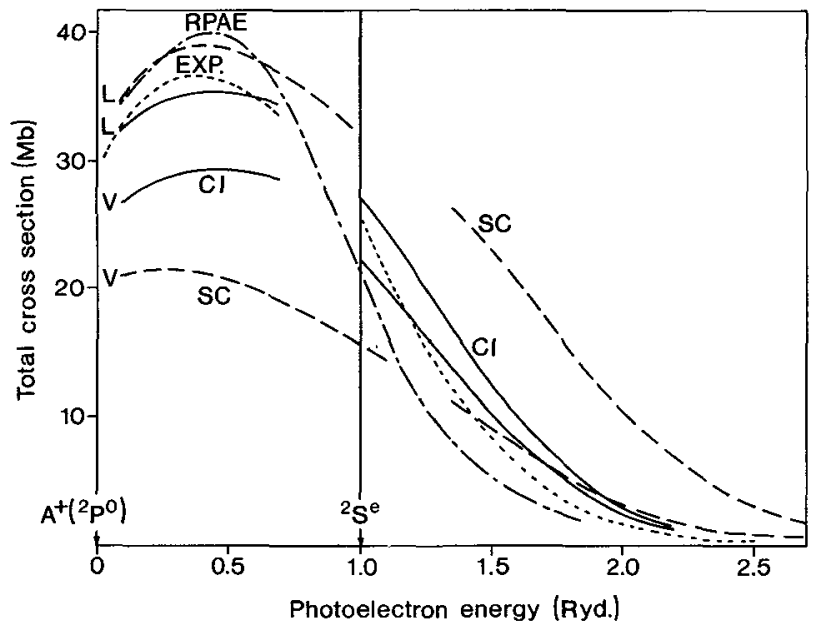

FIG. 8. - The total photoionization cross section of argon.

accord with experiment as are also the R.P.A.E. calculations.

Another quantity which can be measured is the angular distribution parameter $\beta$ of the ejected $3 p$ electron. The $R$-matrix results of Taylor [38] are compared in figure 9 with the experiments of Houlgate et al. [39] and Codling et al. [40] as well as with the R.P.A.E. results of Amusia et al. [41]. Again the C I length results are satisfactory.

We conclude this discussion of argon by showing in figure 10 the partial cross section for the ejection

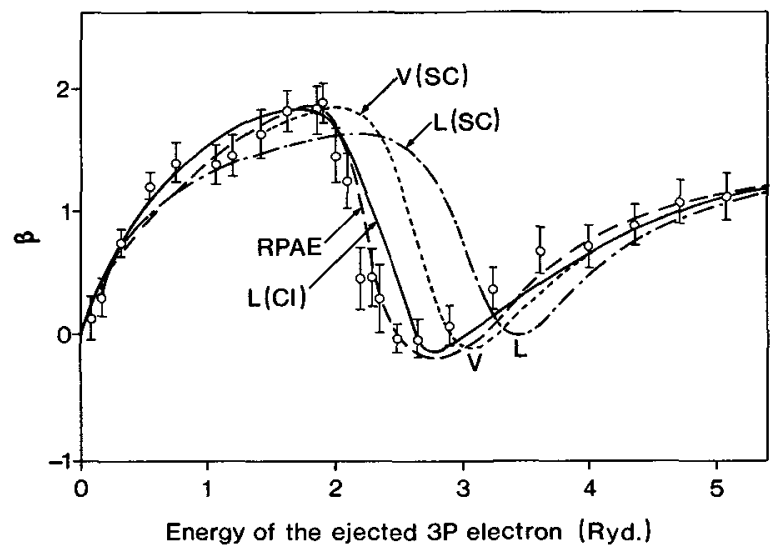

FIG. 9. - Angular distribution parameter $\beta$ for the ejection of the $3 p$ electron in argon.

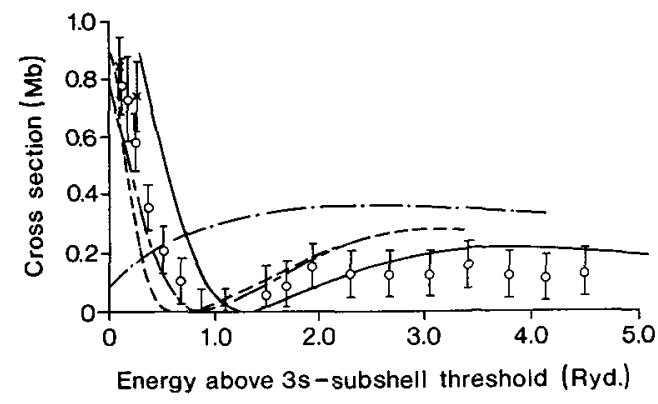

FIG. 10. -- Partial cross section for the photoionization of the 3s electron in argon. Solid curve is the $R$-matrix calculation. of the inner shell $3 \mathrm{~s}$ electron. This channel is strongly effected by the coupling with the other two channels in equation (13) and the $R$-matrix calculation and the R.P.A.E. [42] both give a minimum in the cross section close to threshold which is in good agreement with the experiments of Samson and Gardner [43] and Houlgate et al. [39]. On the other hand the HartreeFock theory of Kennedy and Mansen [44] which neglects this coupling does not show this minimum.

Turning now to open shell atoms we first consider the photoionization of aluminium represented by

$$
\begin{aligned}
& h v+\mathrm{Al}\left(3 \mathrm{~s}^{2} 3 \mathrm{p}^{2} \mathrm{P}^{0}\right) \stackrel{\nearrow}{\rightarrow} \mathrm{Al}^{+}\left(3 \mathrm{~s}^{2}{ }^{1} \mathrm{~S}^{\mathrm{e}}\right)+\mathrm{e}^{-}\left(k \mathrm{~s} 3 \mathrm{p}^{3} \mathrm{P}^{0}\right)+\mathrm{e}^{-}(k \mathrm{~d} k \mathrm{f}) \\
&> \mathrm{Al}^{+}\left(3 \mathrm{~s} 3 \mathrm{p}^{1} \mathrm{P}^{0}\right)+\mathrm{e}^{-}(k \mathrm{p} k \mathrm{f}) .
\end{aligned}
$$

Calculations have been carried out including these three ionic states in expansion (1), where in each of these, virtual excitations into a $\overline{3 \mathrm{~d}}$ pseudo-orbital are included. The total cross section calculated by Le Dourneuf et al. [45] is compared with experiments of Kohl and Parkinson [46] in figure 11. The $R$-matrix

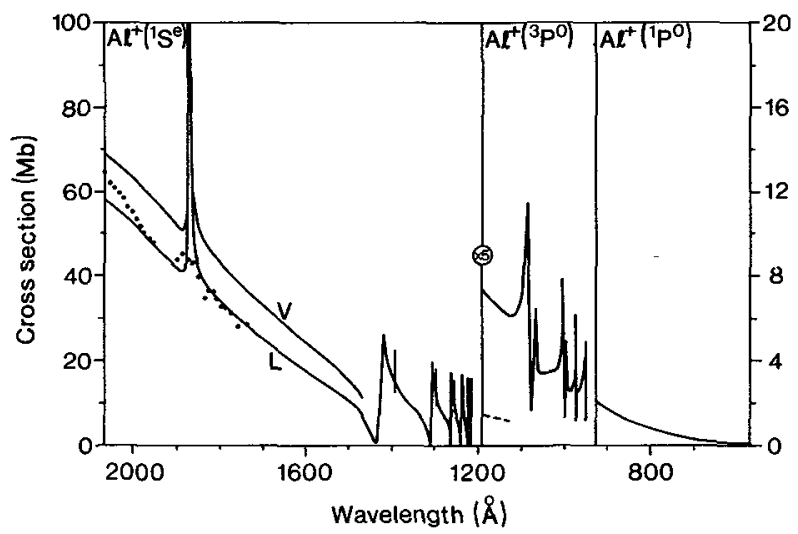

FIG. 11. - The total photoionization cross section of aluminium.

calculation removes a discrepancy between earlier single channel theories by Vainstein et al. [47] and Peach [48] and experiment close to threshold. The broad peak there arises from a $3 \mathrm{~s} 3 \mathrm{p}^{2}{ }^{2} \mathrm{D}^{\mathrm{e}}$ resonance which extendes into the discrete as pointed out by Lin [49]. At higher energies the $R$-matrix calculations are in good agreement with the resonances converging to the higher thresholds as measured by Esteva et al. [50] and Roig [51].

Calculations have also been carried out for the photoionization of the open shell atoms carbon and oxygen [52] and nitrogen [53]. In these cases all ionic states with configurations $2 \mathrm{~s}^{2} 2 \mathrm{p}^{q}$ were included. We show in figure 12 the total photoionization cross section of atomic oxygen compared with the experiments of Cairns and Samson [54]. The agreement is good however the resolution of the experiment is too poor for the resonance series to be observed. 


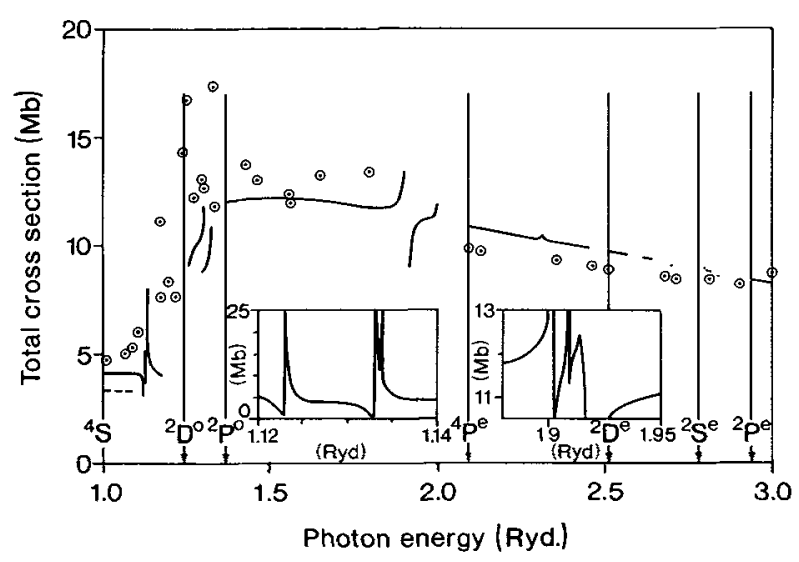

Fig. 12. - The total photoionization cross section of oxygen.

Recent work using the $R$-matrix method has also been reported chlorine [55] where R.P.A.E. calculations are also available [56]. However the final picture for this atom is still nuclear. In addition a number of other open shell atoms are being studied.

4. Future directions and conclusions. - As already mentioned in section 2 the study of electron molecule scattering using the $R$-matrix method is being very actively persued and some results have already been reported for $\mathrm{H}_{2}$ and $\mathrm{N}_{2}$. In addition the extension to treat free-free transitions is well in hand [57].

A major new development will however be needed to treat the situation when there are two or more electrons in the external region as for example occurs for ionization by electron impact. However some initial steps in this direction have been made by Fano and Inokuti [58], and further work in this area can be expected.

In conclusion we believe that the $R$-matrix method is now established as a basic technique for treating a broad range of atomic and molecular collision processes and that many new results will be reported in future years.

Acknowledgments. - The author wishes to acknowledge the many contributions of his co-workers K. L. Bell, K. A. Berrington, P. Dufton, A. E. Kingston, M. Le Dourneuf, I. Mackey, I. Shimamura, W. D. Robb, K. T. Taylor and Vo Ky Lan. He also wishes to thank H. Van Regemorter for the hospitality of his group at the Observatoire de ParisMeudon, section d'astrophysique, where this review was written and where many of the calculations were carried out.

\section{References}

[1] Wigner, E. P.. Phys. Rev. 70 (1946) 15, 606.

[2] Wigner, E. P. and Eisenbud, L., Phys. Rev. 72 (1947) 29.

[3] Lane, A. M. and Thomas, R. G., Rev. Mod. Phys 30 (1958) 257.

[4] Breit, G., Handb. Phys. 41 (1959) 1.

[5] Burke, P. G. and RobB, W. D., Adv. Atom. Molec. Phys. 11 (1975) 143.

16] Le Dourneuf, M. and Vo Ky Lan, J. Phys. B 1- (1977) L35.

[7] Burke, P. G., HibberT, A. and Robb, W. D., J. Phys. B 4 (1971) 153.

[8] Buttle, P. J. A., Phys. Rev. 160 (1967) 719.

[9] Fano, U. and LEe, C. M., Phys. Rev. Lett. 31 (1973) 1573.

[10] LeE, C. M., Phys. Rev. A 10 (1974) 584.

[11] Schneider, B., Chem. Phys. Lett. 31 (1975) 237, Phys. Rev. A 11 (1975) 1957, Phys. Rev. A 14 (1976) 1923.

[12] Schneider. B and Hay, P. J., Phys. Rev. A 13 (1976) 2049

[13] Burke, P. G., Mackey, I. and Shimamura, I., J. Phys. B 10 (1977) 2497

[14] Bloch, C., Nucl. Phys. 4 (1957) 503.

[15] Shimamura, I., Invited paper at the X ICPEAC Paris (1977) Electronic and Atomic Collisions, ed. G. Watel (North Holland) 1978, 213.

[16] Berrington, K. A.. Burke, P. G., Chang, J. J., Chivers, A. T., RoBB, W. D. and TAYlor, K. T., Comput. Phys. Commun. 8 (1974) 149.

[17] Le Dourneuf, M. and Vo Ky Lan, J. Phys. B 1- (1977) L97

Le Dourneuf, M., Thesis (Paris) C.N.R.S. AO 12658 (1976).

[18] Hotop, H. and Lineberger, W. C., J. Chem. Phys. Ref. Data 4 (1975) 539.

[19] Clementi, E. and McClean, A. D., Phys. Rev. 133A (1964) 419.

[20] Moser, C. and Nesbet, R. K., Phys. Rev. A 6 (1972) 1710.

[21] Sasaki, F. and Yoshimine, M., Phys. Rey. A 9 (1974) 17, 26.
[22] Thomas, L. D. and Nesbet, R. K., Phys. Rev. A 11 (1975) 170, A 12 (1975) 2369, 2378.

[23] Miller, T. M., Aubrey, B. B., Eisner, P. N. and Bederson, B., Bull. Am. Phys. Soc. 15 (1970) 416 and private communication.

[24] Neynaber, R. H., Marino, L. L., Rothe, E. W. and Truhllo. S. M., Phys. Rev. 123 (1961) 148, 129 (1963) 2069.

[25] Lin, S. C. and Kivel, B., Phys. Rev. 114 (1959) 1027.

[26] Sunshine, G., Aubrey, B. B. and Bederson, B., Phys. Rev. 154 (1967) I.

[27] Rountree, S. P., Smith, E. R. and Henry, R. J. W., J. Phys. B 7 (1974) L167.

[28] Rовв, W. D., Invited paper at the X ICPEAC Paris (1977) Electronic and Atomic Collisions, ed. G. Watel (North Holland) 1978, 231 .

129] Berrington, K. A., Burke, P. G., Dufton, P. L. and KingsTON. A. E., J. Phys. B 10 (1977) 1465.

[30] EISSNLR, W., The Physits of Electronics and Atomic Collisions. ed. T. R. Govers and F. J. de Heer (Amsterdam, NorthHolland) 1972, 460.

[31] Flower, D. R. and Launay, J. M., Astron. Astrophys. 29 (1973) 321

[32] Dufton, P. L., Berrington, K. A., Burke, P. G. and KingsTON, A. E., Astron. Astrophys., to be published.

[33] Burke, P. G. and Taylor, K. T., J. Phys. B 8 (1975) 2620.

[34] Amusia, M. Ya. and Cherepkov, N. A., Case Stud. At. Phys. 5 (1975) 47.

[35] Kelly, H. P., Adv. Chem. Phys. 14 (1976) 129, Photoiontzation and other probes of many-electrons interaction (Plenum Press) 1976. 83.

[36] Amusia, M. Ya, Cherepkov, N. A. and Chernysheva, L. V., Sov. Phys. J.E.T.P. 33 (1971) 90. 
[37] Marr, G. V. and West, J. B., At. Data (1977) to be published.

[38] TAYloR, K. T., J. Phys. B (1977) to be published.

[39] Houlgate, R. G., West, J. B., Codling, K. and Marr, G. V. J. Phys. B 7 (1974) L470, J. Electron Spectrosc. 9 (1976) 205.

[40] Codling, K., Houlgate, R. G., West, J. B. and Woodruff, P. R., J. Phys. B 9 (1976) L83.

[41] Amusia, M. Ya, Cherepkov, N. A. and Chernysheva, L. V.. Phys. Lett. 40A (1972) 15.

[42] Amusia, M. Ya, Ivanov, V. K., Cherepkov, N. A. and CherNYSHEVA, L. V., Phys. Lett. 40A (1972) 361.

[43] Samson, J. A. R. and Gardner, J. L., Phys. Rev. Lett. 33 (1974) 671.

[44] Kennedy, D. J. and Manson, S. T., Phys. Rev. A 5 (1972) 227.

[45] Le Dourneuf, M., Vo Ky Lan, Burke, P. G. and Taylor. K. T., J. Phys. B 8 (1975) 2640.

[46] Kohl, J. L. and PARkinson, W. H., Astrophys. J. 184 (1973) 641.

[47] Vainstein, L. A. and Norman, G. E., Opt. Spectrosc. 8 (1960) 79.
[48] Peach, G., Mon. Not. R. Astron. Soc, 124 (1962) 371.

[49] Lin, C. D., Astrophys. J. 187 (1974) 385.

[50] Esteva, J. M., Mehlman-Balloft, G. and Romand, J., J. Quant. Spectrosc. Radiat. Transfer 12 (1972) 1291.

[51] Rolg, R. A., J. Phys. B 8 (1975) 2939.

[52] TAYlor, K. and BURKe, P. G., J. Phys. B 9 (1976) L353.

[53] Le Dourneuf, M., Vo Ky Lan and Hibbert, A., J. Phys. B 9 (1976) L359.

[54] Cairns, R. B. and Samson, J. A. R., Phys. Rey. 139 (1965) A1403.

[55] Lamoureux, M. and Combet-Farnoux, F., J. Physique Colloq. 39 (1978) C4.

[56] Starace, A. F. and Armstrong, L. Jr, Phys. Rev. A 13 (1976) 1850.

[57] Bell, K. L., Burke, P. G. and Kingston, A. E., J. Phys. B 10 (1977) 3177.

[58] Fano, U. and Inokuti, M., Argonne Nat. Lab. Report No 7680 (1976). 